\title{
Robust Background Subtraction with Shadow and Highlight Removal for Indoor Surveillance
}

\author{
Jwu-Sheng Hu and Tzung-Min Su \\ Department of Electrical and Control Engineering, National Chiao-Tung University, Hsinchu 300, Taiwan
}

Received 1 March 2006; Revised 12 September 2006; Accepted 29 October 2006

Recommended by Francesco G. B. De Natale

\begin{abstract}
This work describes a robust background subtraction scheme involving shadow and highlight removal for indoor environmental surveillance. Foreground regions can be precisely extracted by the proposed scheme despite illumination variations and dynamic background. The Gaussian mixture model (GMM) is applied to construct a color-based probabilistic background model (CBM). Based on CBM, the short-term color-based background model (STCBM) and the long-term color-based background model (LTCBM) can be extracted and applied to build the gradient-based version of the probabilistic background model (GBM). Furthermore, a new dynamic cone-shape boundary in the RGB color space, called a cone-shape illumination model (CSIM), is proposed to distinguish pixels among shadow, highlight, and foreground. A novel scheme combining the CBM, GBM, and CSIM is proposed to determine the background which can be used to detect abnormal conditions. The effectiveness of the proposed method is demonstrated via experiments with several video clips collected in a complex indoor environment.
\end{abstract}

Copyright (c) 2007 J.-S. Hu and T.-M. Su. This is an open access article distributed under the Creative Commons Attribution License, which permits unrestricted use, distribution, and reproduction in any medium, provided the original work is properly cited.

\section{INTRODUCTION}

Image background subtraction is an essential step in many vision-based home-care applications, especially in the field of monitoring and surveillance. If foreground objects can be precisely extracted through background subtraction, the computing time of the following vision algorithms will be reduced due to limited searching regions and the efficiency becomes better because of neglecting noises outside the foreground regions.

A reference image is generally used to perform background subtraction. The simplest means of obtaining a reference image is by averaging a period of frames [1]. However, it is not suitable to apply time averaging on the homecare applications because the foreground objects (especially for the elderly people or children) usually move slowly and the household scene changes constantly due to light variations from day to night, switches of fluorescent lamps and furniture movements, and so forth. In short, the deterministic methods such as the time averaging have been found to have limited success in practice. For indoor environments, a good background model must also handle the effects of illumination variation, and the variation from background and shadow detection. Furthermore, if the background model cannot handle the fast or slow variations from sunlight or fluorescent lamps, the entire image will be regarded as foreground. That is, a single model cannot represent the distribution of pixels with twinkling values. Therefore, to describe a background pixel by a bimodel instead of a single model is necessary in home-care applications in the real world.

Two approaches were generally adopted to build up a bimodel of background pixel. The first approach is termed the parametric method, and uses single Gaussian distribution [2] or mixtures of Gaussian [3] to model the background image. Attempts were made to improve the GMM methods to effectively design the background model, for example, using an online updated algorithm of GMM [4] and the Kalman filter to track the variation of illumination in the background pixel [5]. The second approach is called the nonparametric method, and uses the kernel function to estimate the density function of background images [6].

Another important consideration is the shadows and highlights. Numerous recent studies have attempted to detect the shadows and highlights. Stockham [7] proposed that a pixel contains both an intensity value and a reflection factor. If a pixel is termed the shadow, then a decadent factor is implied on that pixel. To remove the shadow, the decadent factor should be estimated to calculate the real pixel value. 


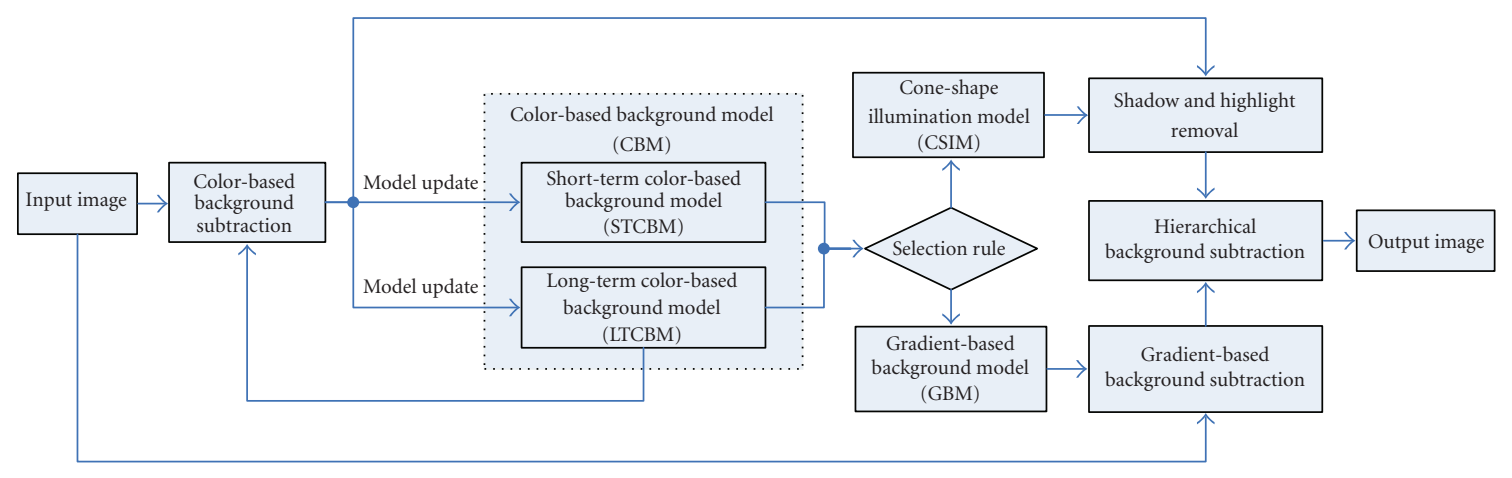

FIGURE 1: Block diagram showing the proposed scheme for background subtraction with shadow removal.

Rosin and Ellis [8] proposed that shadow is equivalent to a semitransparent region, and uses two properties for shadow detection. Moreover, Elgammal et al. [9] tried to convert the RGB color space to the rgb color space (chromaticity coordinate). Because illumination change is insensitive in the chromaticity coordinate, shadows are not considered the foreground. However, lightness information is lost in the rgb color space. To overcome this problem, a measure of lightness is used at each pixel [9]. However, the static thresholds are unsuitable for dynamic environment.

Indoor surveillance applications require solving environmental changes and shadow and highlight effects. Despite the existence of abundance of research on individual techniques, as described above, few efforts have been made to investigate the integration of environmental changes and shadow and highlight effects. The contribution of this work is the scheme to combine the color-based background model (CBM), the gradient-based background model (GBM), and the coneshape illumination model (CSIM). In CSIM, a new dynamic cone-shape boundary in the RGB color space is proposed for efficiently distinguishing a pixel from the foreground, shadow, and highlight. A selection rule combined with the short-term color-based background model (STCBM) and long-term color-based background model (LTCBM) is also proposed to determine the parameters of GBM and CSIM. Figure 1 illustrates the block diagram of the overall scheme.

The remainder of this paper is organized as follows. Section 2 describes the statistical learning method used in the probabilistic modeling and defines STCBM and LTCBM. Section 3 then proposes CSIM using STCBM and LTCBM to classify shadows and highlights efficiently. A hierarchical background subtraction framework that combined with color-based subtraction, gradient-based subtraction, and shadow and highlight removal was then described to extract the real foreground of an image. In Section 4, experimental results are presented to demonstrate the performance of the proposed method in complex indoor environments. Finally, Section 5 presents discussions and conclusions.

\section{BACKGROUND MODELING}

Our previous investigation [10] studied a CBM to record the activity history of a pixel via GMM. However, the foreground regions generally suffer from rapid intensity changes and require a period of time to recover themselves when objects leave the background. In this work, STCBM and LTCBM are defined and applied to improve the flexibility of the gradientbased subtraction that proposed by Javed et al. [11].The features of images used in this work include pixel color and gradient information. This study assumes that the density functions of the color features and gradient features are both Gaussian distributed.

\subsection{Color-based background modeling}

First, each pixel $x$ is defined as a 3-dimensional vector $(R, G, B)$ at time $t$. $N$ Gaussian distributions are used to construct the GMM of each pixel, which is described as follows:

$$
f(x \mid \lambda)=\sum_{i=1}^{N} w_{i} \frac{1}{\sqrt{(2 \pi)^{d}\left|\sum_{i}\right|}} \exp \left(-\frac{1}{2}\left(x-\mu_{i}\right)^{T} \sum_{i}^{-1}\left(x-\mu_{i}\right)\right),
$$

where $\lambda$ represents the parameters of GMM,

$$
\lambda=\left\{w_{i}, \mu_{i}, \sum_{i}\right\}, \quad i=1,2, \ldots, N, \quad \sum_{i=1}^{N} w_{i}=1 .
$$

Suppose $X=\left\{x_{1}, x_{2}, \ldots, x_{m}\right\}$ is defined as a training feature vector containing $m$ pixel values collected from a pixel among a period of $m$ image frames. The next step is calculating the parameter $\lambda$ of GMM of each pixel so that the GMM can match the distribution of $X$ with minimal errors. A common method for calculating $\lambda$ is the maximum likelihood (ML) estimation. ML estimation aims to find model parameters by maximizing the GMM likelihood function. ML parameters can be obtained iteratively using the expectation maximization (EM) algorithm and the maximum likelihood estimation of $\lambda$ is defined as follows:

$$
\lambda_{\mathrm{ML}}=\arg \max _{\lambda} \sum_{j=1}^{m} \log f\left(x_{j} \mid \lambda\right) .
$$


The EM algorithm involves two steps; the parameters of GMM can be derived by iteratively using the expectation step equation and maximum step equation, as follows:

\section{Expectation step (E step):}

$$
\beta_{j i}=\frac{w_{i} f\left(x_{j} \mid \mu_{i}, \sum_{i}\right)}{\sum_{k=1}^{N} a_{k} f\left(x_{j} \mid \mu_{k}, \sum_{k}\right)}, \quad i=1, \ldots, N, j=1, \ldots, m
$$

$\beta_{j i}$ denotes the posterior probability that the feature vector $x_{j}$ belongs to the $i$ th Gaussian component distribution.

\section{Maximum step (M step):}

$$
\begin{gathered}
\widehat{w}_{i}=\frac{1}{N} \sum_{j=1}^{m} \beta_{j i}, \\
\hat{\mu}_{i}=\frac{\sum_{j=1}^{m} \beta_{j i} x_{j}}{\sum_{j=1}^{m} \beta_{j i}}, \\
\widehat{\sum}_{i}=\frac{\sum_{j=1}^{m} \beta_{j i}\left(x_{j}-\hat{\mu}_{i}\right)\left(x_{j}-\hat{\mu}_{i}\right)^{T}}{\sum_{j=1}^{m} \beta_{j i}} .
\end{gathered}
$$

The termination criteria of the EM algorithm are as follows:

(a) the increment between the new log-likelihood value and the last log-likelihood value is below a minimum increment threshold;

(b) The iterative count exceeds a maximum iterative count threshold.

Suppose an image contains total $S=W \times H$ pixels, where $W$ means the image width and $H$ means the image height and then there are total $S$ GMMs should be calculated by the EM algorithm with the collected training feature vector of each pixel.

Moreover, this study uses the $K$-means algorithm [12], which is an unsupervised data clustering used before the EM algorithm iterations to accelerate the convergence. First, $N$ random values are chosen from $X$ and assigned as the center of each class. Then the following steps are applied to cluster the $m$ values of the training feature vector $X$.

(a) Calculate the 1-norm distances between the $m$ values and the $N$ center values. Each value of $X$ is classified to the class which has the minimum distance with it.

(b) After clustering all the values of $X$, recalculate each class center by calculating the mean of the values among each class.

(c) Calculate the 1-norm distances between the $m$ values and the $N$ new center values. Each value of $X$ is classified to the class which has the minimum distance with it. If the new clustering result is the same as the clustering result before recalculating each class center, then stop, otherwise return to previous step to calculate the $N$ new center values.
After applying $K$-means algorithm to cluster the values of $X$, the mean of each class is assigned as the initial value of $\mu_{i}$, the maximum distance among the points of each class is assigned as the initial value of $\sum_{i}$, and the value of $w_{i}$ is initialized as $1 / N$.

\subsection{Model maintenance of LTCBM and STCBM}

According to the above section, an initial color-based probabilistic background model is created using the training feature vector set $X$ with $N$ Gaussian distributions and $N$ is usually defined as 3 to 5 based on the observation over a short period of time $m$. However, when the background changes are recorded over time, it is possible that more different distributions from the original $N$ distributions are observed. If the GMM of each pixel contains only $N$ Gaussian distributions, only $N$ background distributions are reserved and other collected background information is lost and it is not flexible to model the background with only $N$ Gaussian distributions.

To maintain the representative background model and improve the flexibility of the background model simultaneously, an initial LTCBM is defined as the combination of the initial color-based probabilistic background model and extra $N$ new Gaussian distributions (total $2 N$ distributions), an arrangement inspired by the work of [3]. Kaew et al. [3] proposed a method of sorting the Gaussian distributions based on the fitness value $w_{i} / \sigma_{i}\left(\sum_{i}=\sigma_{i}^{2} I\right)$, and extracted a representative model with a threshold value $B_{0}$.

After sorting the first $N$ Gaussian distributions with fitness value, $b(b \leq N)$ Gaussian distributions are extracted with the following criterion:

$$
B=\underset{b}{\arg \min } \sum_{j=1}^{b} w_{j}>B_{0}
$$

The first $b$ Gaussian distributions are defined as the elected color-based background model (ECBM) to be the criterion to determine the background. Meanwhile, the remainders $(2 N-b)$ of the Gaussian distributions are defined as the candidate color-based background model (CCBM) for dealing with the background changes. Finally, LTCBM is defined using the combination of the ECBM and CCBM. Figure 2 shows the block diagram to illustrate the process of building the initial LTCBM, ECBM, and CCBM.

The Gaussian distributions of ECBM mean the characteristic distributions of "background." Therefore, if a new pixel value belongs to any of the Gaussian distributions of ECBM, the new pixel is regarded as "a pixel contains the property of background" and the new pixel is classified as "background." In this work, a new pixel value is considered as background when it belongs to any Gaussian distribution in ECBM and has a probability not exceeding 2.5 standard deviations away from the corresponding distribution. If none of the $b$ Gaussian distributions match the new pixel value, a new test is conducted by checking the new pixel value against the Gaussian distributions in CCBM. The parameters of the Gaussian 


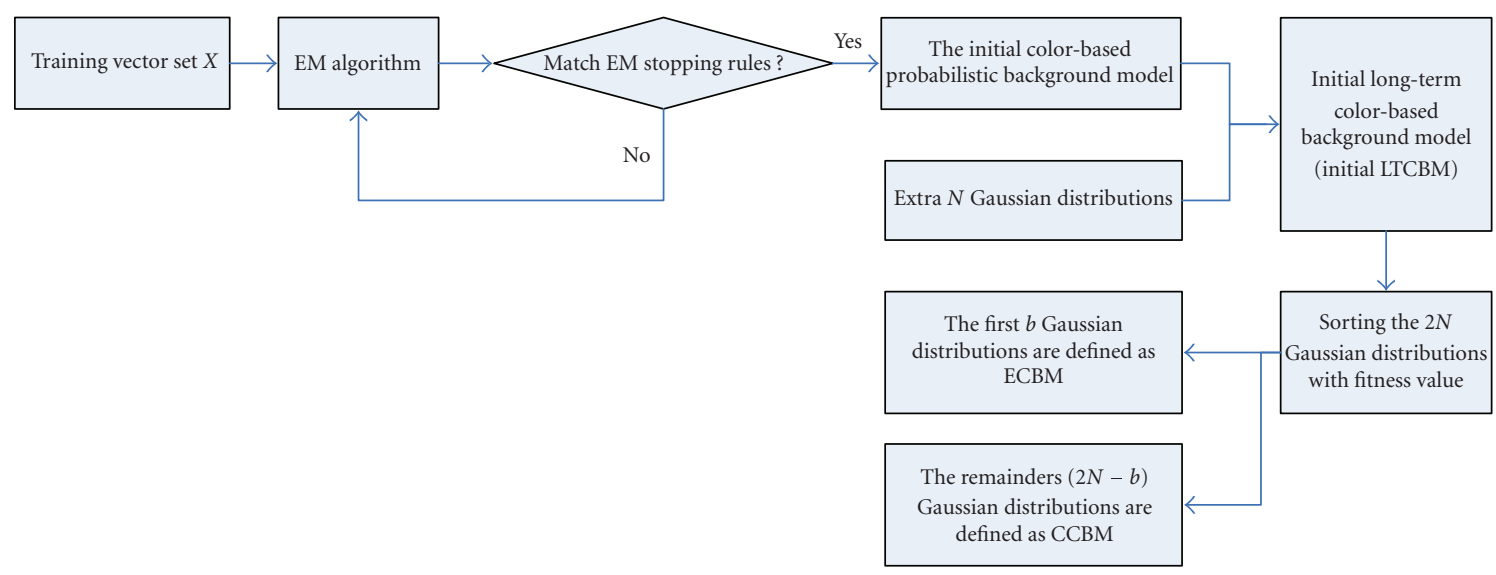

FIGURE 2: Block diagram showing the process of building the initial LTCBM, ECBM and CCBM.

distributions are updated via the following equations:

$$
\begin{gathered}
w_{i}^{t+1}=(1-\alpha) w_{i}^{t}+\alpha \hat{p}\left(w_{i}^{t} \mid X_{i}^{t+1}\right), \\
m_{i}^{t+1}=(1-\rho) m_{i}^{t}+\rho X_{i}^{t+1}, \\
\sum_{i}^{t+1}=(1-\rho) \sum_{i}^{t}+\rho\left(X_{i}^{t+1}-m_{i}^{t+1}\right)^{T}\left(X_{i}^{t+1}-m_{i}^{t+1}\right), \\
\rho=\alpha g\left(X_{i}^{t+1} \mid m_{i}^{t}, \sum_{i}^{t}\right),
\end{gathered}
$$

$\rho$ and $\alpha$ are termed the learning rates and determine the update speed of LTCBM. Moreover, $\hat{p}\left(w_{i}^{t} \mid X_{i}^{t+1}\right)$ results from background subtraction which is set to 1 if a new pixel value belongs to the ith Gaussian distribution. If a new incoming pixel value does not belong to any of the Gaussian distributions in CBM and the number of Gaussian components in CCBM is below $(2 N-b)$, a new Gaussian distribution is added to reserve the new background information with three parameters: the current pixel value as the mean, a large predefined value as the initial variance, and a low predefined value as the weight. Otherwise, the $(2 N-b)$ th Gaussian distribution in CCBM is replaced by the new one. After updating the parameters of the Gaussian components, all Gaussian distributions in CBM are resorted by recalculating the fitness values.

Unlike LTCBM, STCBM is defined to record the background changes during a short period. Suppose $B_{1}$ frames are collected during a short period $B_{1}$ and then $B_{1}$ new incoming pixels for each pixel are collected and defined as a test pixel set $P=\left\{p_{1}, p_{2}, \ldots, p_{q}, \ldots, p_{B_{1}}\right\}$, where $p_{q}$ means the new incoming pixel at time $q$. A test pixel set $P$ is defined and used for calculating the STCBM and a result set $S$ is then defined and calculated by comparing $P$ with LTCBM and is described as (8), where $I_{q}$ means the result after background subtraction, which means the index of Gaussian distribution of the initial LTCBM, $R_{q}$ means the index of resorting result for each Gaussian distribution after each update, and $F_{q}$ means the reset flag of each Gaussian distribution,

$$
\begin{aligned}
S=\{ & S_{1}, S_{2}, \ldots, S_{q}, \ldots, S_{B_{1}}, S_{q}=\left(I_{q}, R_{q}(i), F_{q}(i)\right), \\
& \text { where } 1 \leq I_{q} \leq 2 N, 1 \leq R_{q}(i) \leq 2 N, \\
& \left.F_{q}(i) \in\{0,1\}, 1 \leq i \leq 2 N\right\} .
\end{aligned}
$$

The histogram of CG is then given using the following equation:

$$
\begin{gathered}
H_{\mathrm{CG}}(k)=\frac{\sum_{k}\left[\delta\left(k-\left(I_{q}+R_{q}\left(I_{q}\right)\right)\right)+\bar{F}_{q} \cdot \sum_{q^{\prime}} \delta\left(k-\left(I_{q^{\prime}}+R_{q^{\prime}}\left(I_{q}\right)\right)\right)\right]}{B_{1}}, \\
1 \leq k \leq 2 N, \quad 1 \leq q \leq B_{1}, \quad 1 \leq q^{\prime}<q .
\end{gathered}
$$

In brief, four Gaussian distributions are used to explain how (8)-(9) work and the corresponding example is listed in Table 1. At first, the original CBM contains four Gaussian distributions $(2 N=4)$, and the index of Gaussian distribution in the initial CBM is fixed $(1,2,3,4)$. At the first time, a new incoming pixel which belongs to the second Gaussian distribution compares with the CBM, so the result of background subtraction is $I_{q}=2$. Moreover, the CBM is updated with (7) and the index of Gaussian distribution in CBM is changed. When the order of the first and second Gaussian distributions is changed, $R_{q}(i)$ records the change states; for example, $R_{q}(1)=1$ means the first Gaussian distribution has moved forward to the second one, and $R_{q}(2)=-1$ means the second Gaussian distribution has moved backward to the first one. At the second time, a new incoming pixel which belongs to the second Gaussian distribution based on the initial CBM is classified as the first Gaussian distribution $\left(I_{q}=1\right)$ based on the latest order of CBM. However, the CG histogram can be calculated according to the original index of the initial CBM with the latest order of CBM and $R_{q}(i)$, such that $H_{\mathrm{CG}}\left(I_{q}+F_{q}=2\right)$ will be accumulated with one. Moreover, $R_{q}(i)$ changes while the order of Gaussian distributions changes. For example, at the fifth time in Table 1, the order of CBM changes from $(2,1,3,4)$ to $(1,2,3,4)$, and then $R_{q}(1)=1-1=0$ means the first Gaussian distribution 
TABLE 1: The example to calculate CG histogram.

\begin{tabular}{|c|c|c|c|c|c|c|c|c|c|c|c|c|}
\hline Time $(q)$ & $\begin{array}{l}\text { Index of ir } \\
\text { CBM }\end{array}$ & itial & 1 & 2 & 3 & 4 & Time $(q)$ & $\begin{array}{l}\text { Index of initial } \\
\text { CBM }\end{array}$ & 1 & 2 & 3 & 4 \\
\hline \multirow{6}{*}{1} & \multicolumn{2}{|c|}{$\begin{array}{l}\text { Index of CCBM } \\
\text { at time } q\end{array}$} & 1 & 2 & 3 & 4 & \multirow{6}{*}{4} & $\begin{array}{l}\text { Index of CCBM } \\
\text { at time } q\end{array}$ & 2 & 1 & 3 & 4 \\
\hline & \multicolumn{2}{|l|}{$p_{q}$} & - & $*$ & - & - & & $p_{q}$ & $*$ & - & - & - \\
\hline & \multicolumn{2}{|l|}{$I_{q}$} & \multicolumn{4}{|c|}{2} & & $I_{q}$ & \multicolumn{4}{|c|}{2} \\
\hline & \multicolumn{2}{|l|}{$R_{q}$} & 0 & 0 & 0 & 0 & & $R_{q}$ & 1 & -1 & 0 & 0 \\
\hline & \multicolumn{2}{|l|}{$F_{q}$} & 0 & 0 & 0 & 0 & & $F_{q}$ & 0 & 0 & 0 & 0 \\
\hline & \multicolumn{2}{|l|}{ CG } & 0 & 1 & 0 & 0 & & CG & 2 & 2 & 0 & 0 \\
\hline \multirow{6}{*}{2} & \multicolumn{2}{|c|}{$\begin{array}{l}\text { Index of CCBM } \\
\text { at time } q\end{array}$} & 2 & 1 & 3 & 4 & \multirow{6}{*}{5} & $\begin{array}{l}\text { Index of CCBM } \\
\text { at time } q\end{array}$ & 1 & 2 & 3 & 4 \\
\hline & \multicolumn{2}{|l|}{$p_{q}$} & - & $*$ & - & - & & $p_{q}$ & $*$ & - & - & - \\
\hline & \multicolumn{2}{|l|}{$I_{q}$} & \multicolumn{4}{|c|}{1} & & $I_{q}$ & \multicolumn{4}{|c|}{1} \\
\hline & \multicolumn{2}{|l|}{$R_{q}$} & 1 & -1 & 0 & 0 & & $R_{q}$ & 0 & 0 & 0 & 0 \\
\hline & \multicolumn{2}{|l|}{$F_{q}$} & 0 & 0 & 0 & 0 & & $F_{q}$ & 0 & 0 & 0 & 0 \\
\hline & \multicolumn{2}{|l|}{ CG } & 0 & 2 & 0 & 0 & & CG & 3 & 2 & 0 & 0 \\
\hline \multirow{6}{*}{3} & \multicolumn{2}{|c|}{$\begin{array}{l}\text { Index of CCBM } \\
\text { at time } q\end{array}$} & 2 & 1 & 3 & 4 & \multirow{6}{*}{6} & $\begin{array}{l}\text { Index of CCBM } \\
\text { at time } q\end{array}$ & 1 & 2 & 3 & 4 \\
\hline & \multicolumn{2}{|l|}{$p_{q}$} & $*$ & - & - & - & & $p_{q}$ & - & - & $*$ & - \\
\hline & \multicolumn{2}{|l|}{$I_{q}$} & \multicolumn{4}{|c|}{2} & & $I_{q}$ & \multicolumn{4}{|c|}{3} \\
\hline & \multicolumn{2}{|l|}{$R_{q}$} & 1 & -1 & 0 & 0 & & $R_{q}$ & 0 & 0 & 0 & 0 \\
\hline & \multicolumn{2}{|l|}{$F_{q}$} & 0 & 0 & 0 & 0 & & $F_{q}$ & 0 & 0 & 0 & 0 \\
\hline & \multicolumn{2}{|l|}{ CG } & 1 & 2 & 0 & 0 & & CG & 3 & 2 & 1 & 0 \\
\hline & Test pixel $P_{q}$ & $\mathrm{Col}$ & $\begin{array}{l}\text { d background } \\
\text { raction }\end{array}$ & & $\begin{array}{l}\text { The result } \\
\text { backgro }\end{array}$ & $\begin{array}{l}\text { ture } S_{q} \\
\text { iubtract }\end{array}$ & & $\begin{array}{l}\text { ord } S_{q} \text { into the } \\
\text { ult structure } S\end{array}$ & & Calc & & \\
\hline & & & CBM & & $\begin{array}{r}\text { Resortin } \\
\text { distributio }\end{array}$ & $\begin{array}{l}\text { Gauss } \\
\text { f the LT }\end{array}$ & & & & & & \\
\hline
\end{tabular}

FIGURE 3: Block diagram showing the process to calculate $H_{\mathrm{CG}}$ (the histogram of $I_{q}$ ).

of initial CBM has moved back to the first one of the latest $\mathrm{CBM}$, and $R_{q}(2)=-1+1=0$ means the second Gaussian distribution has moved back to the second one of the latest CBM.

If a new incoming pixel $p_{q}$ matches the $i$ th Gaussian distribution that has the least fitness value, the $i$ th Gaussian distribution is replaced with a new one and the flag $F_{q}$ will be set to 1 to reset the accumulated value of $H_{\mathrm{CG}}(i)$. Figure 3 shows the block diagram about the process of calculating $H_{\mathrm{CG}}$.

After matching all test pixels to the corresponding Gaussian distribution, the result set $S$ can be used to calculating $H_{\mathrm{CG}}$ using $I_{q}$ and $F_{q}$. With the reset flag $F_{q}$, STCBM can be built up rapidly based on a simple idea, threshold on the occurring frequency of Gaussian distribution. That is to say, the short-term tendency of background changes is apparent if an element of $H_{\mathrm{CG}}(k)$ is above a threshold value $B_{2}$ during a period of frames $B_{1}$. In this work, $B_{1}$ is assigned a value of 300 frames and $B_{2}$ is set to be 0.8 . Therefore, the representative background component in the short-term tendency can be determined to be $k$ if the value of $H_{\mathrm{CG}}(k)$ exceeds 0.8 , otherwise, STCBM provides no further information on background model selection.

\subsection{Gradient-based background modeling}

Javed et al. [11] developed a hierarchical approach that combines color and gradient information to solve the problem about rapid intensity changes. Javed et al. [11] adopted the $k$ th, highest weighted Gaussian component of GMM at each pixel to obtain the gradient information to build the 
gradient-based background model. The choice of $k$ in [11] is similar to selecting $k$ based only on ECBM defined in this work. However, choosing the highest weighted Gaussian component of GMM leads to the loss of the short term tendencies of background changes. Whenever a new Gaussian distribution is added into the background model, it is not selected owing to its low weighting value for a long period of time. Consequently, the accuracy of the gradient-based background model is reduced for that the gradient information is not suitable for representing the current gradient information.

To solve this problem, both STCBM and LTCBM are considered in selecting the value of $k$ for developing a more robust gradient-based background model and maintaining the sensitivity to short-term changes. When STCBM provides a representative background component (says the $k_{S}$ th bin in STCBM), $k$ is set to $k_{S}$ rather than the highest weighted Gaussian distribution.

Let $x_{i, j}^{t}=[R, G, B]$ be the latest color value that matched the $k_{S}$ th distribution of LTCBM at pixel location $(i, j)$, then the gray value of $x_{i, j}^{t}$ is applied to calculate the gradient-based background subtraction. Suppose the gray value of $x_{i, j}^{t}$ is calculated as (10), then $g_{i, j}^{t}$ will be distributed as (11) based on independence among RGB color channels,

$$
\begin{gathered}
g_{i, j}^{t}=\alpha R+\beta G+\gamma B, \\
g_{i, j}^{t} \sim N\left(m_{i, j}^{t},\left(\sigma_{i, j}^{t}\right)^{2}\right),
\end{gathered}
$$

where

$$
\begin{gathered}
m_{i, j}^{t}=\alpha \mu_{i, j}^{t, k_{s}, R}+\beta \mu_{i, j}^{t, k_{s}, G}+\gamma \mu_{i, j}^{t, k_{s}, B} \\
\sigma_{i, j}^{t}=\sqrt{\alpha^{2}\left(\sigma_{i, j}^{t, k_{s}, R}\right)^{2}+\beta^{2}\left(\sigma_{i, j}^{t, k_{s}, G}\right)^{2}+\gamma^{2}\left(\sigma_{i, j}^{t, k_{s}, B}\right)^{2}} .
\end{gathered}
$$

After that, the gradient along the $x$ axis and $y$ axis can be defined as $f_{x}=g_{i+1, j}^{t}-g_{i, j}^{t}$ and $f_{y}=g_{i, j+1}^{t}-g_{i, j}^{t}$. From the work of [11], $f_{x}$ and $f_{y}$ have the distributions defined in (13),

$$
\begin{aligned}
& f_{x} \sim N\left(m_{f_{x}},\left(\sigma_{f_{x}}\right)^{2}\right), \\
& f_{y} \sim N\left(m_{f_{y}},\left(\sigma_{f_{y}}\right)^{2}\right),
\end{aligned}
$$

where

$$
\begin{gathered}
m_{f_{x}}=m_{i+1, j}^{t}-m_{i, j}^{t}, \\
m_{f_{y}}=m_{i, j+1}^{t}-m_{i, j}^{t}, \\
\sigma_{f_{x}}=\sqrt{\left(\sigma_{i+1, j}^{t}\right)^{2}+\left(\sigma_{i, j}^{t}\right)^{2}}, \\
\sigma_{f_{y}}=\sqrt{\left(\sigma_{i, j+1}^{t}\right)^{2}+\left(\sigma_{i, j}^{t}\right)^{2}} .
\end{gathered}
$$

Suppose $\Delta_{m}=\sqrt{f_{x}^{2}+f_{y}^{2}}$ is defined as the magnitude of the gradient for a pixel, $\Delta_{d}=\sqrt{\tan ^{-1}\left(f_{x} / f_{y}\right)}$ is defined as its direction (the angle with respect to the horizontal axis), and $\Delta=\left[\Delta_{m}, \Delta_{d}\right]$ is defined as the feature vector for modeling the gradient-based background model. The gradient-based background model based on feature vector $\Delta=\left[\Delta_{m}, \Delta_{d}\right]$ can be defined as (15),

$$
\begin{aligned}
& F^{k}\left(\Delta_{m}, \Delta_{d}\right) \\
& \quad=\frac{\Delta_{m}}{2 \prod \sigma_{f_{x}}^{k} \sigma_{f_{y}}^{k} \sqrt{1-\rho^{2}}} \exp \left(-\frac{z}{2\left(1-\rho^{2}\right)}\right)>T_{g},
\end{aligned}
$$

where

$$
\begin{gathered}
z=\left(\frac{\Delta_{m} \cos \Delta_{d}-\mu_{f_{x}}}{\sigma_{f_{x}}}\right)^{2}-2 \rho\left(\frac{\Delta_{m} \cos \Delta_{d}-\mu_{f_{x}}}{\sigma_{f_{x}}}\right) \\
\times\left(\frac{\Delta_{m} \sin \Delta_{d}-\mu_{f_{y}}}{\sigma_{f_{y}}}\right)+\left(\frac{\Delta_{m} \sin \Delta_{d}-\mu_{f_{y}}}{\sigma_{f_{y}}}\right)^{2}, \\
\rho=\frac{\left(\sigma_{i, j}^{t}\right)^{2}}{\sigma_{f_{x}} \sigma_{f_{y}}} .
\end{gathered}
$$

\section{BACKGROUND SUBTRACTION WITH SHADOW REMOVAL}

This section describes shadow and highlight removal, and proposes a framework that combines CBM, GBM, and CSIM to improve background subtraction efficiency.

\subsection{Shadow and highlight removal}

Besides foreground and background, shadows and highlights are two important phenomena that should be considered in most cases. Shadows and highlights result from changes in illumination. Compared with the original pixel value, shadow has similar chromaticity but lower brightness, and highlight has similar chromaticity but higher brightness. The regions influenced by illumination changes are classified as the foreground if shadow and highlight removal is not performed after background subtraction.

Hoprasert et al. [13] proposed a method of detecting highlight and shadow by gathering statistics from $N$ color background images. Brightness and chromaticity distortion are used with four threshold values to classify pixels into four classes. The method that used the mean value as the reference image in [13] is not suitable for dynamic background. Furthermore, the threshold values are estimated based on the histogram of brightness distortion and chromaticity distortion with a given detection rate, and are applied to all pixels regardless of the pixel values. Therefore, it is possible to classify the darker pixel value as shadow. Furthermore, it cannot record the history of background information.

This paper proposes a 3D cone model that is similar to the pillar model proposed by Hoprasert et al. [13], and combines LTCBM and STCBM to solve the above problems. A cone model is proposed with the efficiency in deciding the parameters of $3 \mathrm{D}$ cone model according to the proposed LTCBM and STCBM. In the RGB space, a Gaussian distribution of the LTCBM becomes an ellipsoid whose center is the mean of the Gaussian component, and the length of each principle axis equals 2.5 standard deviations of the Gaussian component. A new pixel $I(R, G, B)$ is considered to belong 

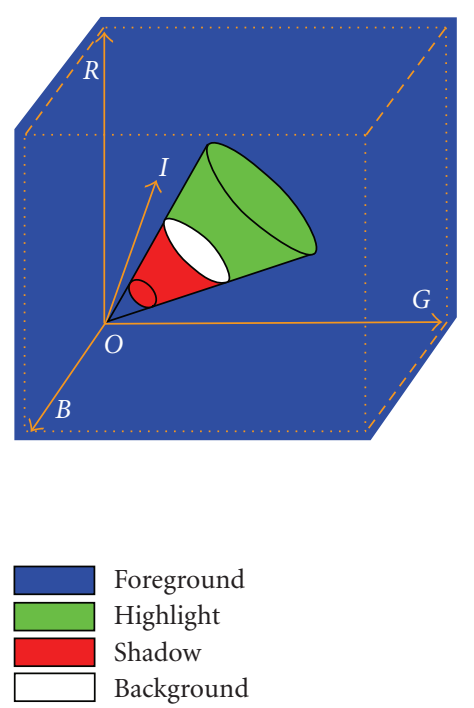

FIGURE 4: The proposed 3D cone model in the RGB color space.

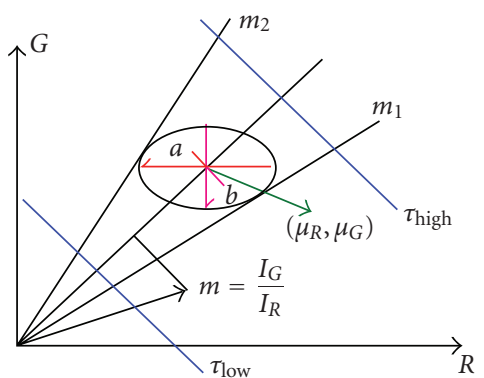

FIGURE 5: 2D projection of the 3D cone model from RGB space onto the RG space.

to background if it is located inside the ellipsoid. The chromaticities of the pixels located outside the ellipsoid but inside the cone (formed by the ellipsoid and the origin) resemble the chromaticity of the background. The brightness difference is then applied to classify the pixel as either highlight or shadow. Figure 4 illustrates the 3D cone model in the RGB color space.

The threshold values $\alpha_{\text {low }}$ and $\alpha_{\text {high }}$ are applied to avoid classifying the darker pixel value as shadow or the brighter value as highlight, and can be selected based on the standard deviation of the corresponding Gaussian distribution in CBM. Because the standard deviations of the $R, G$, and $B$ color axes are different, the angles between the curved surface and the ellipsoid center are also different. It is difficult to classify the pixel using the angles in the $3 \mathrm{D}$ space. The $3 \mathrm{D}$ cone is projected onto the $2 \mathrm{D}$ space to classify a pixel using the slope and the point of tangency. Figure 5 illustrates the projection of the $3 \mathrm{D}$ cone model onto the RG $2 \mathrm{D}$ space.

Let $a$ and $b$ denote the lengths of major and minor axis of the ellipse, where $a=2.5 * \sigma_{R}$ and $b=2.5 * \sigma_{G}$. The center of the ellipse is $\left(\mu_{R}, \mu_{G}\right)$, and the elliptical equation is described as (17),

$$
\frac{\left(R-\mu_{R}\right)^{2}}{a^{2}}+\frac{\left(G-\mu_{G}\right)^{2}}{b^{2}}=1 .
$$

The line $G=m R$ is assumed to be the tangent line of the ellipse with the slope $m$. Equation (11) can then be solved using the line equation $G=m R$ with (18),

$$
m_{1,2}=\frac{-\left(2 \mu_{R} \mu_{G}\right) \pm \sqrt{\left(a^{2}-\mu_{R}^{2}\right)^{2}-4\left(2 \mu_{R} \mu_{G}\right)\left(b^{2}-\mu_{G}^{2}\right)}}{2\left(a^{2}-\mu_{R}^{2}\right)} .
$$

A matching result set is given by $F_{b}=\left\{f_{b i}, i=1,2,3\right\}$, where $f_{b i}$ is the matching result of a specific $2 \mathrm{D}$ space. A pixel vector $I=\left[I_{R}, I_{G}, I_{B}\right]$ is then projected onto the $2 \mathrm{D}$ spaces of $R-G, G-B$, and $B-R$. The pixel matching result is set to 1 when the slope of the projected pixel vector is between $m_{1}$ and $m_{2}$. Meanwhile, if the background mean vector is $E=$ $\left[\mu_{R}, \mu_{G}, \mu_{B}\right]$, the brightness distortion $\alpha_{b}$ can be calculated via (19),

$$
\alpha_{b}=\frac{\|I\| \cos (\theta)}{\|E\|}
$$

where

$$
\theta=\left|\theta_{I}-\theta_{E}\right|=\left|\tan ^{-1}\left(\frac{I_{G}}{\sqrt{I_{R}^{2}+I_{B}^{2}}}\right)-\tan ^{-1}\left(\frac{\mu_{G}}{\sqrt{\mu_{R}^{2}+\mu_{B}^{2}}}\right)\right| .
$$

The image pixel is classified as highlight, shadow, or foreground using the matching result set $F_{b}$, the brightness distortion $\alpha_{b}$ and (21),

$$
\mathrm{C}(i)= \begin{cases}\text { Shadow, } & \sum F_{b}=3, \tau_{\text {low }}<\alpha_{b}<1, \text { else, } \\ \text { Highlight, } & \sum F_{b}=3,1<\alpha_{b}<\tau_{\text {high }}, \text { else, } \\ \text { Foreground, } & \text { otherwise. }\end{cases}
$$

When a pixel is a large standard deviation away from a Gaussian distribution, the Gaussian distribution probability of the pixel approximately equals to zero. It also means the pixel does not belong to the Gaussian distribution. By using the simple concept, $\tau_{\text {high }}$ and $\tau_{\text {low }}$ can be chosen using $N_{G}$ standard deviation of the corresponding Gaussian distribution in CBM and are described as (22),

$$
\begin{gathered}
\tau_{\text {high }}=1+\frac{\|S\| \cdot \cos \theta_{\tau}}{\|E\|}, \\
\tau_{\text {low }}=1-\frac{\|S\| \cdot \cos \theta_{\tau}}{\|E\|},
\end{gathered}
$$

where

$$
\begin{gathered}
\|E\|=\sqrt{\left(\mu_{R}\right)^{2}+\left(\mu_{G}\right)^{2}+\left(\mu_{B}\right)^{2}}, \\
\|S\|=\sqrt{\left(N_{G} \cdot \sigma_{R}\right)^{2}+\left(N_{G} \cdot \sigma_{G}\right)^{2}+\left(N_{G} \cdot \sigma_{B}\right)^{2}}, \\
\theta_{\tau}=\left|\theta_{E}-\theta_{S}\right|=\left|\tan ^{-1}\left(\frac{\mu_{G}}{\sqrt{\mu_{R}^{2}+\mu_{B}^{2}}}\right)-\tan ^{-1}\left(\frac{\sigma_{G}}{\sqrt{\sigma_{R}^{2}+\sigma_{B}^{2}}}\right)\right| .
\end{gathered}
$$




\subsection{Background subtraction}

A hierarchical approach combining color-based background subtraction and gradient-based background subtraction has been proposed by Javed et al. [11]. This work proposes a similar method for extracting the foreground pixels. Given a new image frame $I$, the color-based background model is set to LTCBM and STCBM, and gradient-based model is $F^{k}\left(\Delta_{m}, \Delta_{d}\right) . C(I)$ is defined as the result of color-based background subtraction using CBM. $G(I)$ is defined as the result of gradient-based background subtraction. $C(I)$ and $G(I)$ can be extracted by testing every pixel of frame $I$ using the LTCBM and $F^{k}\left(\Delta_{m}, \Delta_{d}\right)$. Moreover, $C(I)$ and $G(I)$ are both defined as a binary image, where 1 represents the foreground pixel and 0 represents the background pixel. The foreground pixels labeled in $C(I)$ are further classified as shadow, highlight, and foreground by using the proposed $3 \mathrm{D}$ cone model. $C(I)$ can then be obtained from $C(I)$ after transferring the foreground pixels which have been labeled as shadow and highlight in $C(I)$ into the background pixel. The difference between Javed et al. [11] and the proposed method is that a pixel classifying procedure using CSIM is applied before using the connected component algorithm to group all the foreground pixels in $C(I)$. The robustness of background subtraction is enhanced due to the better accuracy in $\left|\partial R_{a}\right|$. Moreover, the foreground pixels can be extracted using (24),

$$
\frac{\sum_{(i, j) \in \partial R_{a}}(\nabla I(i, j) G(i, j))}{\left|\partial R_{a}\right|} \geq P_{B},
$$

where $\nabla I$ denotes the edges of image $I$ and $\partial R_{a}$ represents the number of boundary pixels of region $R_{a}$.

\section{EXPERIMENTAL RESULTS}

The video data for experiments was obtained using a SONY DVI-D30 PTZ camera in an indoor environment. Morphological filter was applied to remove noise and the camera controls were set to automatic mode. The same threshold values were used for all experiments. The values of the important threshold values were $N_{G}=15, \alpha=0.002, P_{B}=0.1$, $B_{0}=0.7, B_{1}=300$, and $B_{2}=0.8$. Meanwhile, the computational speed was around five frames per second on a $\mathrm{P} 4$ $2.8 \mathrm{GHz}$ PC, while the video had a frame size of $320 \times 240$.

\subsection{Experiments for local illumination changes}

The first experiment was performed to test the robustness of the proposed method about the local illumination changes. Local illumination changes resulting from desk lights occur constantly in indoor environments. Desk lights are usually white or yellow. Two video clips containing several changes of desk light are collected to simulate local illumination changes. Figure 6(a) shows 15 representative samples of the first one video clip. Meanwhile, Figure 6(b) shows the classified result of the foreground pixel using the proposed method, CBM and CSIM, where red indicates shadow, green indicates highlight, and blue indicates fore- ground. Figure 6(c) displays the result of the final background subtraction to demonstrate the robustness of the proposed method, where the white and black color represents the foreground and background pixels, respectively. The image sequences comprise different levels of illumination changes. The desk light was turned on at the 476th frame and its brightness increased until the 1000th frame. The overall picture becomes the foreground regions of the corresponding frames in Figure 6(b) owing to the lack of such information in CBM. However, the final result of background subtraction of the corresponding frames in Figure 6(c) is still good owing to the proposed scheme combining CBM, CSIM, and GBM. The desk light was then turned off at the 1030th frame and became darker until the 1300th frame. The original Gaussian distribution in the ECBM became the component in CCBM, and a new representative Gaussian distribution in ECBM is constructed for that a new background information is involved from the new collected frames between the 476th and the 1000th frame are more than the initial collected 300 frames. Consequently, the 1300th frame in Figure 6(b) has many foreground regions. However, the final result of the 1300th frame is still good. The illumination changes are all modeled into LTCBM when the background model records the background changes. The area of the red, blue, and green regions reduces after the 1300th frame.

Table 2 compares the proposed scheme with the method proposed by Hoprasert et al. [13]. Comparison criteria are identified by labeling the foreground regions of a frame manually. CSIM can be constructed based on the appropriate representative Gaussian distribution chosen from LTCBM and STCBM. The ability to handle illumination variation and the accuracy of the background subtraction are improved and the results are shown in Table 2.

Figure 7 (a) shows a similar image sequence to that on Figure 6(a). The two sequences differ only in the color of the desk light. The desk light was turned on at the 660th frame and the same brightness was maintained until the 950th frame. The desk light was then turned off at the 1006th frame and turned on again at the 1180th frame. The results of shadows and highlights removal are shown in Figure 7(b) and the results of final background subtraction are shown in Figure $7(\mathrm{c})$. The results of background subtraction in Figure 7 and the comparison result in Table 3 are shown to demonstrate the robustness of the proposed scheme.

\subsection{Experiments for global illumination changes}

The second experiment was performed to test the robustness of the proposed method in terms of global illumination changes. The image sequences consist of illumination changes where a fluorescent lamp was turned on at the 381th frame and more lamps were turned on at the 430th frame. The illumination changes are then modeled into LTCBM when the proposed background model recorded the background changes. Notably the area of the red, blue, and green regions decreases at the 580th frame. When the third daylight lamp is switched on in the 650th frame, it is clear that fewer 


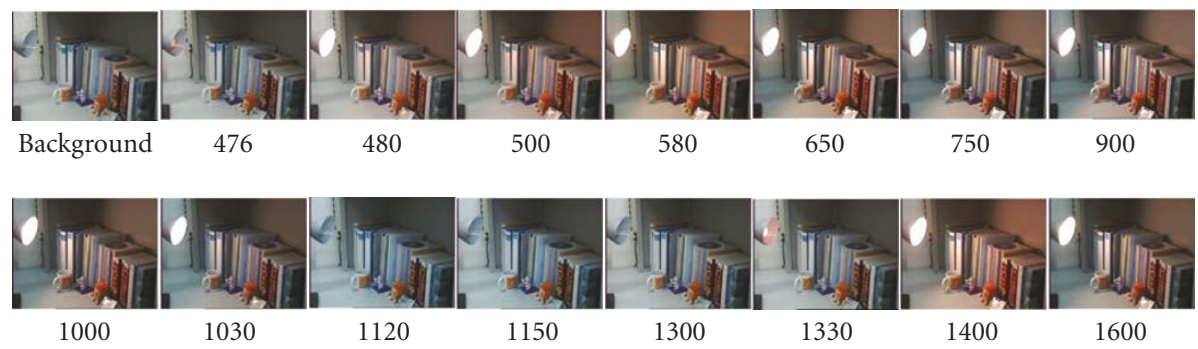

(a)

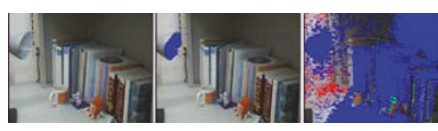

Background 476

480

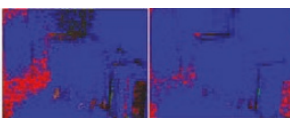

500

580
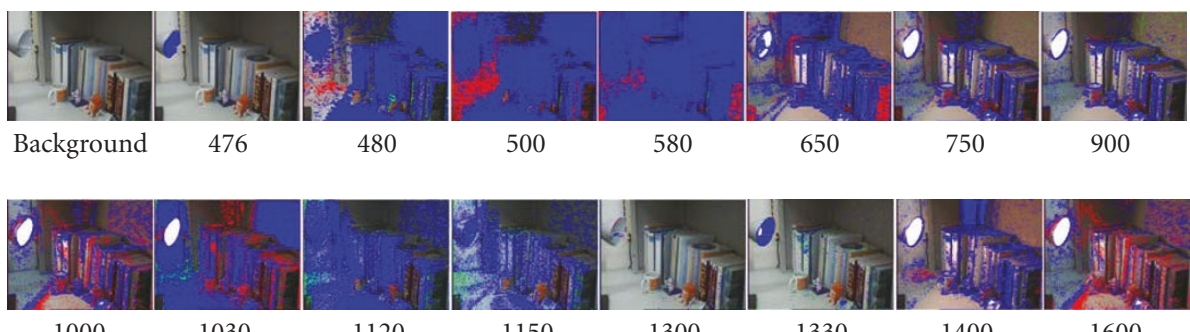

(b)
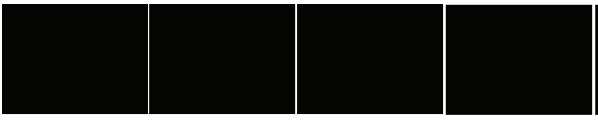

Background

476

480

500

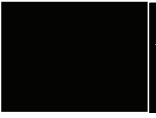

580
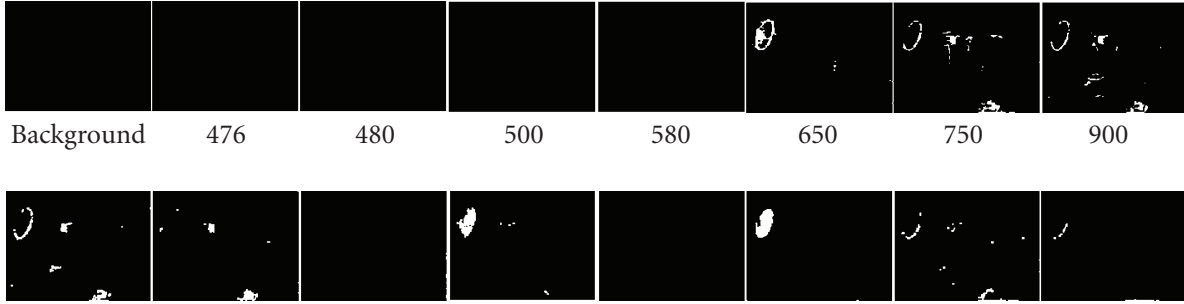

1030

1120

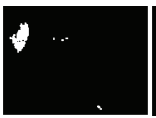

1150
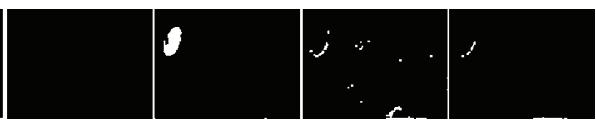

1330

1400

1600

(c)

FIGURE 6: The results of illumination changes with a yellow desk light, the number below the picture is the index of frame, (a) original images, (b) the results of pixel classification, where red indicates the shadow, green indicates the highlight, and blue indicates the foreground, (c) the results of background subtraction with shadow removal using the proposed method, where dark indicates the background and white indicates the foreground.

TABLE 2: The robustness test between the proposed method and that proposed by Hoprasert et al. [13] via local illumination changes with a yellow desk light.

\begin{tabular}{|c|c|c|c|c|c|c|}
\hline \multicolumn{2}{|r|}{ Frame } & 476 & 480 & 500 & 580 & 650 \\
\hline Proposed $(\% *)$ & Hoprasert et al. [13] $\left(\%^{*}\right)$ & $100.00 \quad 94.05$ & $99.84 \quad 36.40$ & $99.93 \quad 22.50$ & $99.91 \quad 15.38$ & $83.96 \quad 23.42$ \\
\hline & Frame & 750 & 900 & 1000 & 1030 & 1120 \\
\hline Proposed $(\% *)$ & Hoprasert et al. [13] $(\% *)$ & $91.50 \quad 31.51$ & $93.10 \quad 30.91$ & $95.44 \quad 34.26$ & $97.75 \quad 38.28$ & $99.15 \quad 32.90$ \\
\hline & Frame & 1150 & 1300 & 1330 & 1400 & 1600 \\
\hline Proposed $(\% *)$ & Hoprasert et al. [13] $\left(\%^{*}\right)$ & 93.79 & 99.95 & 93.3192 .40 & 96.22 & 99.30 \\
\hline
\end{tabular}

* The value in the table means the recognition rate that correct background pixels in a frame divide total pixels in a frame (\%).

blue regions appear at the 845th frame owing to illumination changes having been modeled in the LTCBM. However, the final results of background subtraction shown in Figure 8(c) are all better than those of pure color-based background subtraction shown in Figure 8(b). Table 4 shows the comparison results between the proposed scheme and that proposed by Hoprasert et al. [13]. The comparison demonstrates that the proposed scheme is robust to global illumination changes.

\subsection{Experiments for foreground detection}

In the third experiment (Figure 9), a person goes into the monitoring area, and the foreground region can be effectively extracted regardless of the influence of shadow and highlight in the indoor environment. Owing to the captured video clip having little illumination variation and dynamic background variation, the comparison of the recognition rate of final background subtraction between the proposed method 


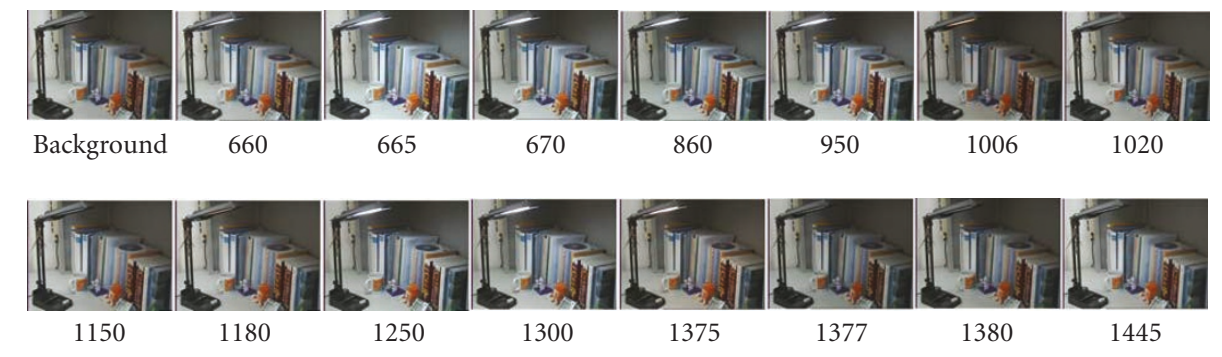

(a)

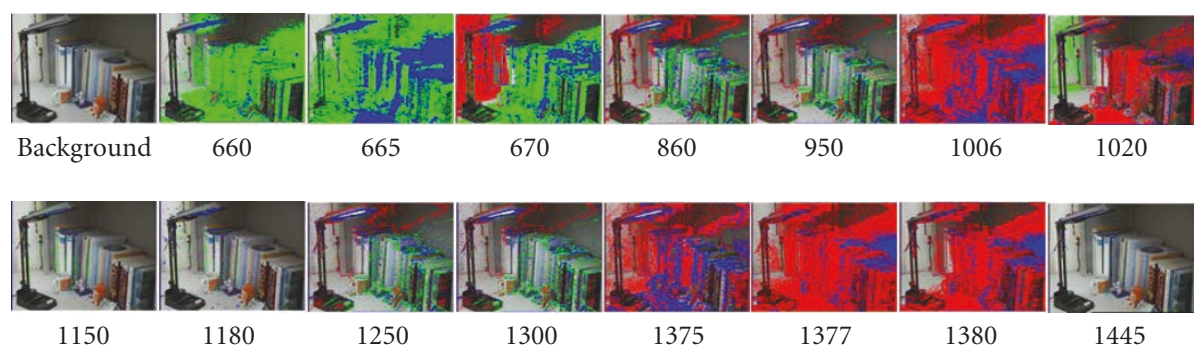

(b)

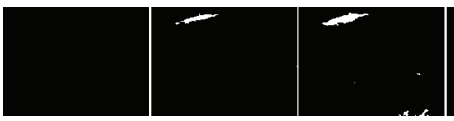

Background

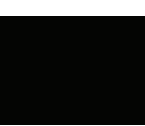

1150

660

665

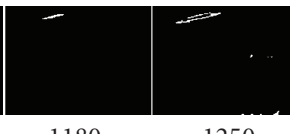

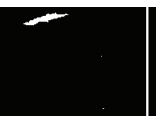

670

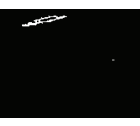

860

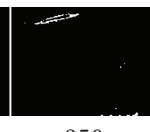

950

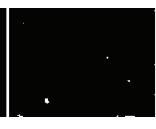

1006

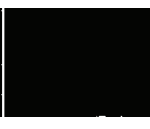

1020
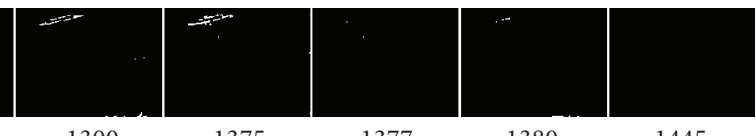

(c)

FIGURE 7: The results of illumination changes with white desk light, the number below the picture is the index of frame, (a) original images, where red indicates the shadow, green indicates the highlight, and blue indicates the foreground, (b) the results of pixel classification, (c) the results of background subtraction with shadow removal using our proposed method, where dark indicates the background and white indicates the foreground.

TABLE 3: The robustness test between the proposed method and that proposed by Hoprasert et al. [13] via local illumination changes with a white desk light.

\begin{tabular}{|c|c|c|c|c|c|c|c|c|c|c|c|}
\hline \multicolumn{2}{|r|}{ Frame } & \multicolumn{2}{|c|}{660} & \multicolumn{2}{|c|}{665} & \multicolumn{2}{|c|}{670} & \multicolumn{2}{|c|}{860} & \multicolumn{2}{|c|}{950} \\
\hline Proposed $(\% *)$ & Hoprasert et al. [13] $(\% *)$ & 99.02 & 99.48 & 97.93 & 79.81 & 95.92 & 92.22 & 96.73 & 93.81 & 97.44 & 94.46 \\
\hline & Frame & \multicolumn{2}{|c|}{ - } & \multicolumn{2}{|c|}{1020} & \multicolumn{2}{|c|}{1150} & \multicolumn{2}{|c|}{1180} & \multicolumn{2}{|c|}{1250} \\
\hline Proposed $(\% *)$ & Hoprasert et al. [13] $(\% *)$ & 98.12 & 95.65 & 99.94 & 98.85 & 99.78 & 99.68 & 98.94 & 99.08 & 97.28 & 93.81 \\
\hline & Frame & \multicolumn{2}{|c|}{ - } & \multicolumn{2}{|c|}{1375} & \multicolumn{2}{|c|}{1377} & \multicolumn{2}{|c|}{1380} & \multicolumn{2}{|c|}{1445} \\
\hline Proposed $(\% *)$ & Hoprasert et al. [13] (\%*) & 97.49 & 95.26 & 97.73 & 87.50 & 98.83 & 98.92 & 99.73 & 99.32 & 100.00 & 99.71 \\
\hline
\end{tabular}

* The value in the table means the recognition rate that correct background pixels in a frame divide total pixels in a frame (\%).

and that of Hoprasert et al. [13] reveals that both methods are about the same, as listed in Table 5.

\subsection{Experiments for dynamic background}

In the fourth experiment (Figure 10), image sequences consist of swaying clothes hung on a frame. The proposed method gradually recognizes the clothes as background owing to the ability of LTCBM to record the history of background changes. In situations involving large variation of dynamic background, a representative initial color-based background model can be established by using more training frames to handle the variations.

\subsection{Experiments for short-term color-based background model}

The final experiment (Figure 11) shows the advantage of adding STCBM. A doll is placed on the desk at the 360th frame. Initially, it is regarded as foreground, and at the 560th 


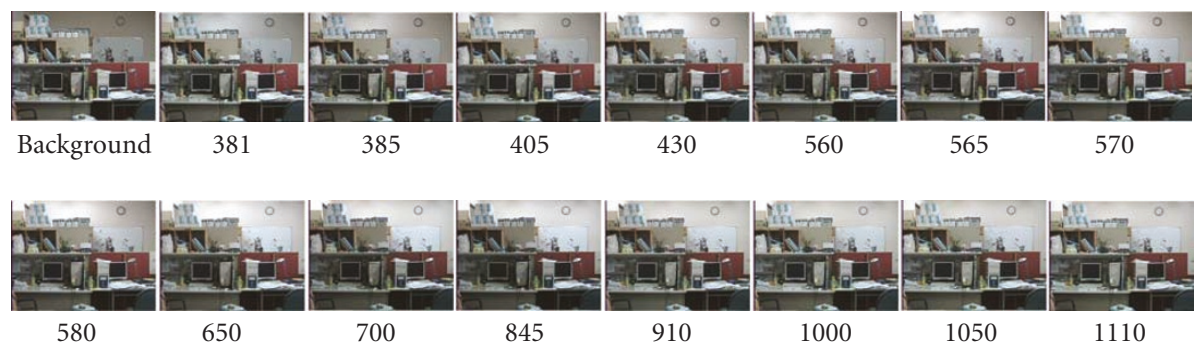

(a)

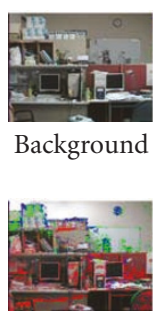

580

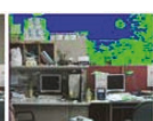

381

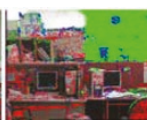

650

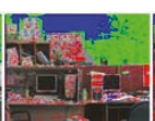

385

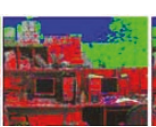

405

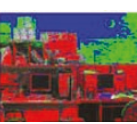

430

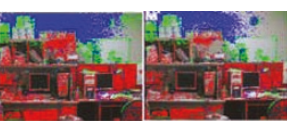

560

565

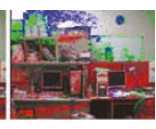

570

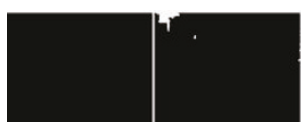

Background

381

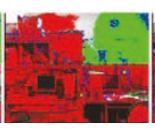

700

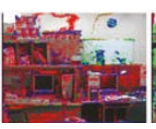

845

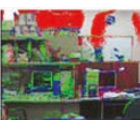

910
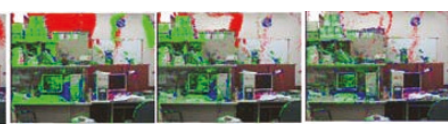

1000

1050

1110

(b)

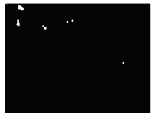

580

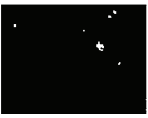

650

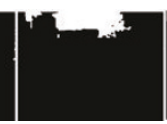

385

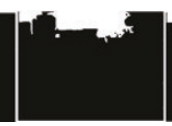

405

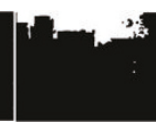

430

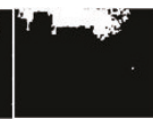

560

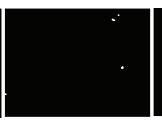

1000

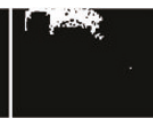

565

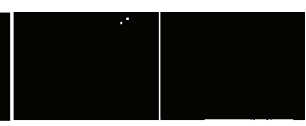

1050

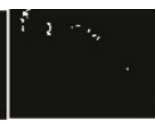

570

1110

(c)

FIGURE 8: The results of global illumination changes with fluorescent lamps, the number below the picture is the index of frame, (a) original images, (b) the results of pixel classification, where red indicates the shadow, green indicates the highlight, and blue indicates the foreground, (c) the results of background subtraction with shadow removal using our proposed method, where dark indicates the background and white indicates the foreground.

TABLE 4: The comparison between the proposed method and that proposed by Hoprasert et al. [13] via global illumination changes with fluorescent lamps.

\begin{tabular}{|c|c|c|c|c|c|c|c|c|c|c|c|}
\hline \multicolumn{2}{|r|}{ Frame } & \multicolumn{2}{|c|}{$381\left(1^{* *}\right)$} & \multicolumn{2}{|c|}{$385\left(1^{* *}\right)$} & \multicolumn{2}{|c|}{$405\left(1^{* *}\right)$} & \multicolumn{2}{|c|}{$430\left(2^{* *}\right)$} & \multicolumn{2}{|c|}{$560\left(2^{* *}\right)$} \\
\hline Proposed $(\% *)$ & Hoprasert et al. [13] $\left(\%^{*}\right)$ & 98.24 & 93.54 & 88.35 & 82.14 & 83.85 & 78.24 & 56.50 & 68.42 & 66.85 & 69.82 \\
\hline & Frame & \multicolumn{2}{|c|}{$565\left(2^{* *}\right)$} & \multicolumn{2}{|c|}{$570\left(2^{* *}\right)$} & \multicolumn{2}{|c|}{$580\left(2^{* *}\right)$} & \multicolumn{2}{|c|}{$650\left(3^{* *}\right)$} & \multicolumn{2}{|c|}{$700\left(3^{* *}\right)$} \\
\hline Proposed $(\% *)$ & Hoprasert et al. [13] $(\% *)$ & 79.87 & 69.30 & 96.88 & 69.69 & 99.08 & 69.55 & 99.23 & 45.62 & 99.49 & 46.22 \\
\hline & Frame & \multicolumn{2}{|c|}{$845\left(3^{* *}\right)$} & \multicolumn{2}{|c|}{$910\left(3^{* *}\right)$} & \multicolumn{2}{|c|}{$1000\left(3^{* *}\right)$} & \multicolumn{2}{|c|}{$1050\left(3^{* *}\right)$} & \multicolumn{2}{|c|}{$1110(3 v)$} \\
\hline Proposed $(\% *)$ & Hoprasert et al. [13] $\left(\%^{*}\right)$ & 99.56 & 46.18 & 99.39 & 53.58 & 99.85 & 57.87 & 99.93 & 60.83 & 99.64 & 60.32 \\
\hline
\end{tabular}

* The value in the table means the recognition rate that correct background pixels in a frame divide total pixels in a frame (\%).

** The number inside the parentheses indicates the number of fluorescent lamps that have turned on.

frame, the foreground region becomes background owing to the LTCBM. However, the Gaussian component belonging to the doll still does not have the highest weighting. Without adding STCBM, when a hand is placed above the doll at the 590th frame, the foreground regions at the 670th frame remain the same as those at the 590th frame, as shown in Figure 11(b). The foreground regions under our hand become shadows at the 670th frame in Figure 11(c) for that shadows and highlights removal works well using a representative Gaussian component based on STCBM. This experiment demonstrates the efficiency of STCBM that a representative Gaussian component of CBM can be selected by giving consideration to long-term tendency and short-term tendency. Besides, the advantage of STCBM helps to reduce the computing time used in GBM and increase the recognition rate of foreground detection. 


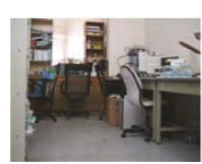

Background

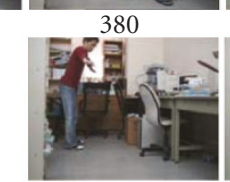

680

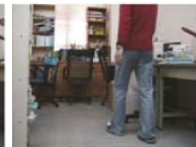

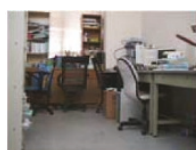

Background

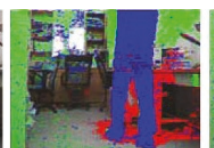

380

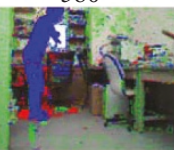

680

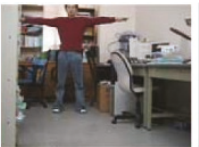

450

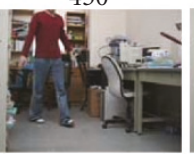

700
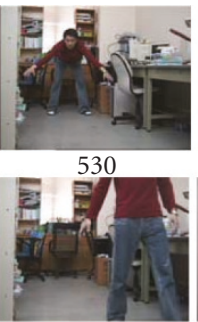

735

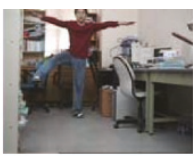

590

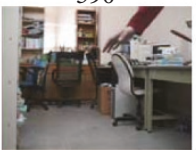

755
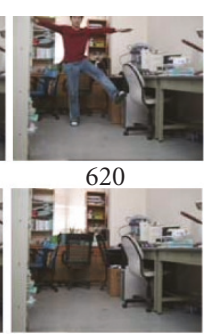

840 (a)
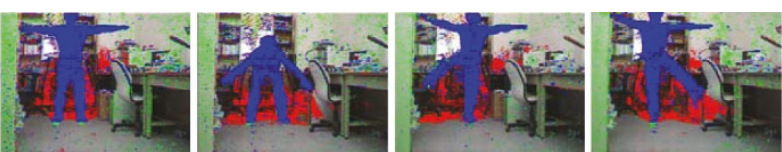

450

530

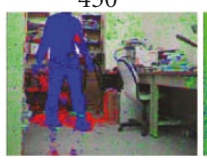

700

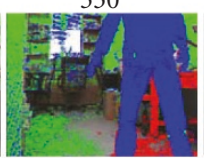

735

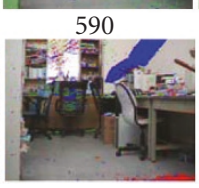

755

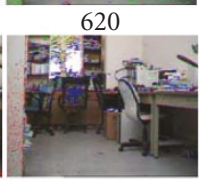

840

(b)
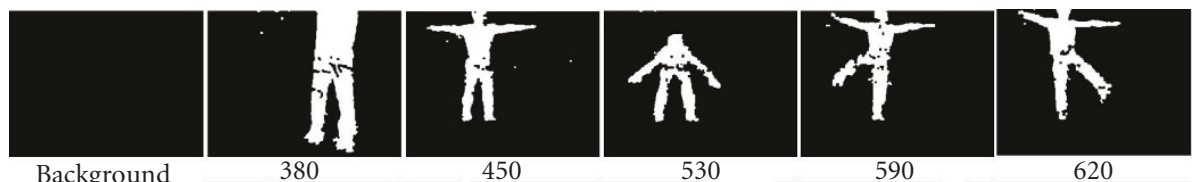

Background

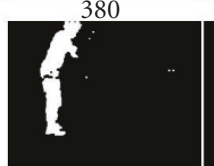

680

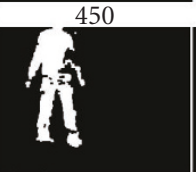

700

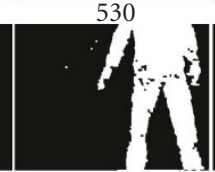

735

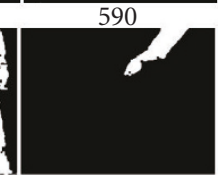

755

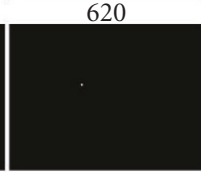

840

(c)

FIGURE 9: The results of foreground detection, (a) original images, (b) the results of pixel classification, where the red color indicates the shadow, green indicates the highlight, and blue indicates the foreground, (c) the results of background subtraction with shadow removal using our proposed method, where dark indicates the background and white indicates the foreground.

TABLE 5: The comparison between the proposed method and that proposed by Hoprasert et al. [13] via foreground detection.

\begin{tabular}{|c|c|c|c|c|c|c|c|c|c|c|c|}
\hline \multicolumn{2}{|r|}{ Frame } & \multicolumn{2}{|c|}{380} & \multicolumn{2}{|c|}{450} & \multicolumn{2}{|c|}{530} & \multicolumn{2}{|c|}{590} & \multicolumn{2}{|c|}{620} \\
\hline Proposed $(\% *)$ & Hoprasert et al. [13] $(\% *)$ & 90.45 & 89.18 & 86.50 & 85.80 & 89.38 & 88.87 & 88.45 & 87.72 & 88.67 & 88.76 \\
\hline & Frame & \multicolumn{2}{|c|}{680} & \multicolumn{2}{|c|}{700} & \multicolumn{2}{|c|}{735} & \multicolumn{2}{|c|}{755} & \multicolumn{2}{|c|}{840} \\
\hline Proposed $(\% *)$ & Hoprasert et al. [13] (\%*) & 91.07 & 90.62 & 85.63 & 85.15 & 82.76 & 80.71 & 92.44 & 92.46 & 100.00 & 99.61 \\
\hline
\end{tabular}

* The value in the table means the recognition rate that correct background pixels in a frame divide total pixels in a frame (\%).

\section{CONCLUSIONS}

This work addressed the problem of subtracting the background from an input image using three models, namely, the color-based background model (CBM), gradient-based background model (GBM), and cone-shape illumination model (CSIM). In the CBM, the elected color-based background model (ECBM), and candidate color-based model (CCBM) are defined to increase the ability of recording a long period of background changes. Short-term color-based background model (STCBM) and long-term color-based background model (LTCBM) are defined to improve the flexibility and robustness of the gradient-based background subtraction. Most important, CSIM is proposed to extract the shadow, and highlight in this paper with a $3 \mathrm{D}$ cone-shape boundary and combined with CBM in the RGB color space. The threshold values $\tau_{\text {high }}$ and $\tau_{\text {low }}$ of CSIM can be calculated automatically using the standard deviation of the Gaussian distribution selected using STCBM and LTCBM. The proposed 3D cone model is compared with the nonparametric model in a complex indoor environment. The experimental results show the effectiveness of the proposed scheme for background subtraction with shadow and highlight removal. 


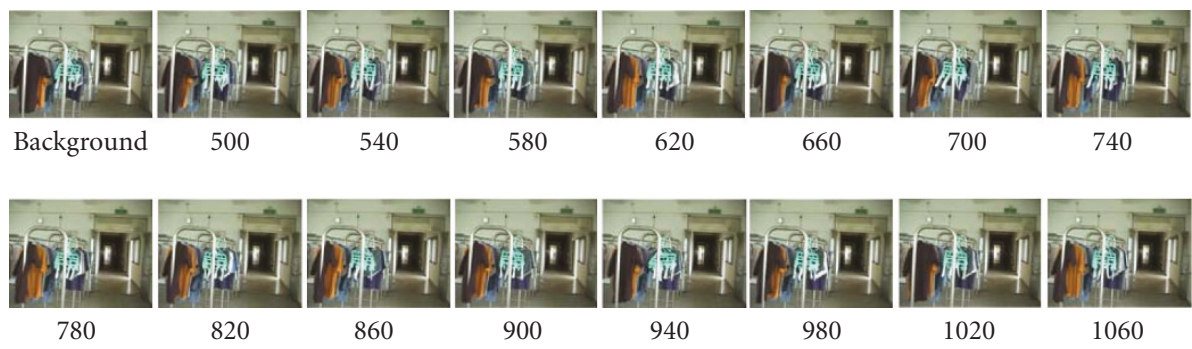

(a)

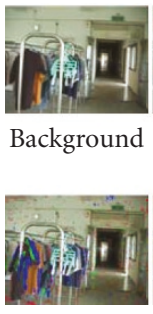

780

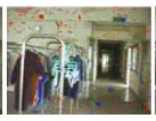

500

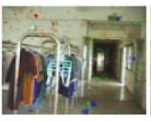

540

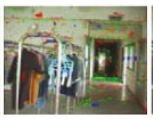

580

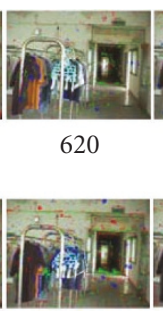

940

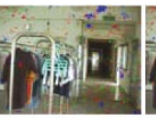

660

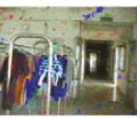

700

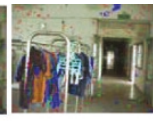

740

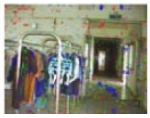

820

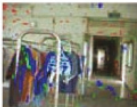

860

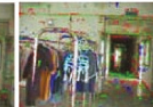

900

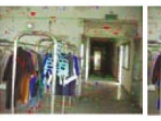

980

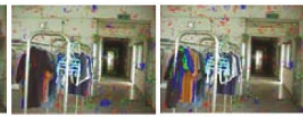

1020
1060

(b)

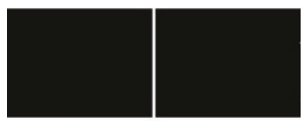

Background

500

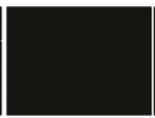

540

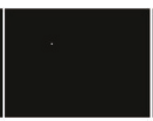

580

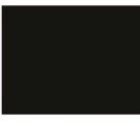

620

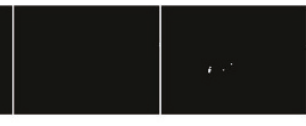

660

700

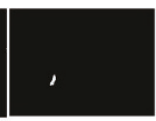

740

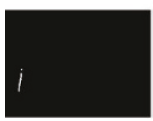

780

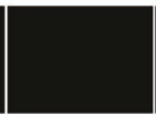

820

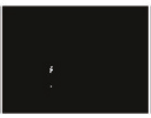

860

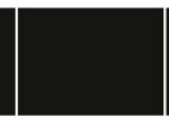

900

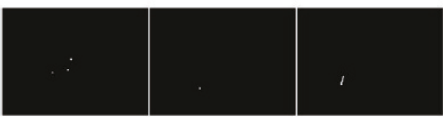

940

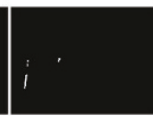

1060

(c)

FIGURE 10: The results of background subtraction about dynamic background, (a) original images, (b) the results of pixel classification, where red color indicates the shadow, green indicates the highlight, and blue indicates the foreground, (c) the results of background subtraction with shadow removal using our proposed method, where dark indicates the background and white indicates the foreground.

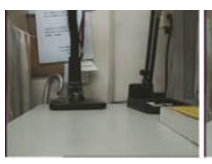

301

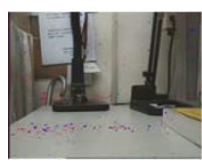

301

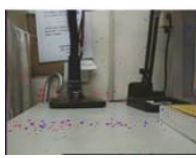

301

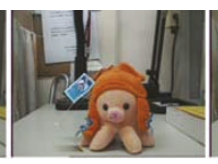

360

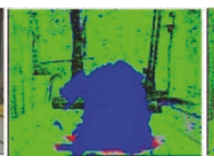

360

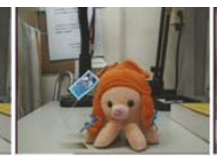

560

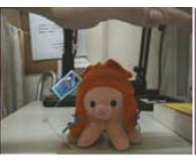

590

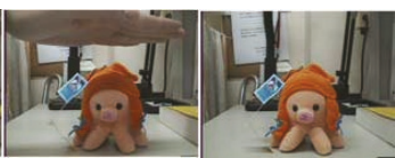

670

(a)

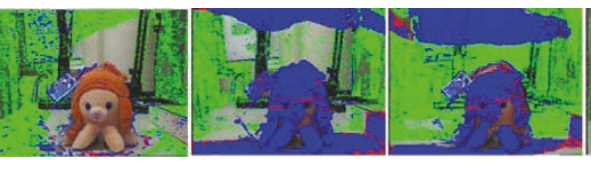

590

(b)

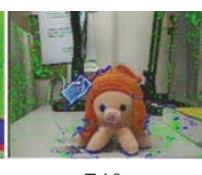

740

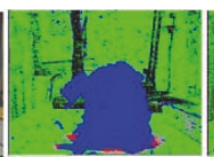

360

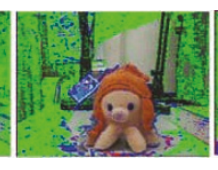

560

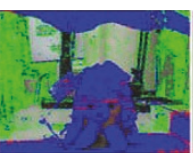

590

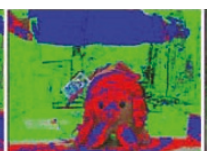

670

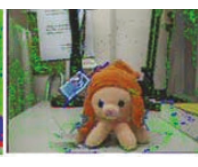

740

(c)

FIGURE 11: The results of the advantage of STCBM, where the red color means the shadow, the green color means the highlight, and the blue color means the foreground, (a) original images, (b) the results of background subtraction without STCBM, (c) the results of background subtraction with STCBM, where red indicates the shadow, green indicates the highlight, and blue indicates the foreground. 


\section{ACKNOWLEDGMENTS}

This work was supported by National Science Council of the ROC under Grant no. NSC93-2218-E009064 and MOE ATU Program under the account number 95W803E.

\section{REFERENCES}

[1] N. Friedman and S. Russell, "Image segmentation in video sequences: a probabilistic approach," in Proceedings of the 13th Conference Uncertainty in Artificial Intelligence (UAI '97), pp. 175-181, Providence, RI, USA, August 1997.

[2] C. R. Wren, A. Azarbayejani, T. Darrell, and A. P. Pentland, "Pfinder: real-time tracking of the human body," IEEE Transactions on Pattern Analysis and Machine Intelligence, vol. 19, no. 7, pp. 780-785, 1997.

[3] P. Kaew, P. Trakul, and R. Bowden, "An improved adaptive background mixture model for real-time tracking with shadow detection," in Proceedings of the 2nd European Workshop on Advanced Video-Based Surveillance Systems, Kingston, UK, September 2001.

[4] C. Stauffer and W. E. L. Grimson, "Adaptive background mixture models for real-time tracking," in Proceedings of the IEEE Computer Society Conference on Computer Vision and Pattern Recognition, vol. 2, pp. 246-252, Fort Collins, Colo, USA, June 1999.

[5] D. Koller, J. Weber, T. Huang, et al., "Towards robust automatic traffic scene analysis in real-time," in Proceedings of the 33rd IEEE Conference on Decision and Control, vol. 4, pp. 37763781, Lake Buena Vista, Fla, USA, December 1994.

[6] A. Elgammal, R. Duraiswami, D. Harwood, and L. S. Davis, "Background and foreground modeling using nonparametric kernel density estimation for visual surveillance," Proceedings of the IEEE, vol. 90, no. 7, pp. 1151-1163, 2002.

[7] T. G. Stockham Jr., "Image processing in the context of a visual model," Proceedings of the IEEE, vol. 60, no. 7, pp. 828-842, 1972.

[8] P. L. Rosin and T. Ellis, "Image difference threshold strategies and shadow detection," in Proceedings of the 6th British $\mathrm{Ma}$ chine Vision Conference, pp. 347-356, Birmingham, Ala, USA, September 1995.

[9] A. Elgammal, D. Harwood, and L. S. Davis, "Non-parametric Model for Background Subtraction," in Proceedings of the 6th European Conference on Computer Vision, pp. 751-767, Dublin, Ireland, June 2000.

[10] T.-M. Su and J.-S. Hu, "Background removal in vision servo system using Gaussian mixture model framework," in Proceeding of IEEE International Conference on Networking, Sensing and Control, vol. 1, pp. 70-75, Taipei, Taiwan, March 2004.

[11] O. Javed, K. Shafique, and M. Shah, "A hierarchical approach to robust background subtraction using color and gradient information," in Proceedings of IEEE Workshop on Motion and Video Computing (MOTION '02), pp. 22-27, Orlando, Fla, USA, December 2002.

[12] J. B. MacQueen, "Some methods for classification and analysis of multivariate observations," in Proceedings of the 5th Berkeley Symposium on Mathematical Statistics and Probability, vol. 1, pp. 281-297, Berkeley, Calif, USA, 1967.

[13] T. Hoprasert, D. Harwood, and L. S. Davis, "A statistical approach for real-time robust background subtraction and shadow detection," in Proceedings of the 7th IEEE International Conference on Computer Vision, Frame Rate Workshop (ICCV'99), pp. 1-19, Kerkyra, Greece, September 1999.
Jwu-Sheng $\mathbf{H u}$ received the B.S. degree from the Department of Mechanical Engineering, National Taiwan University, Taiwan, in 1984, and the M.S. and Ph.D. degrees from the Department of Mechanical Engineering, University of California at Berkeley, in 1988 and 1990, respectively. He is currently a Professor in the Department of Electrical and Control Engineering, $\mathrm{Na}$ tional Chiao-Tung University, Taiwan. His current research interests include microphone array signal processing, active noise control, intelligent mobile robots, embedded systems and applications.

Tzung-Min Su was born in 1978. He received the B.S. degree in electrical and control engineering from National Chiao-Tung University, Taiwan, in 2000. He is currently a Ph.D. candidate in Department of Electrical and Control Engineering at National Chiao-Tung University, Taiwan. He is the Championship of TI DSP Solutions Design Challenge in 2000 and of the national competition held by Ministry of Education Ad-

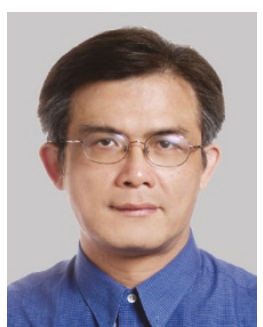
visor Office in 2001. His research interests include background subtraction, 3D object recognition, home-care surveillance, and mobile robot localization.

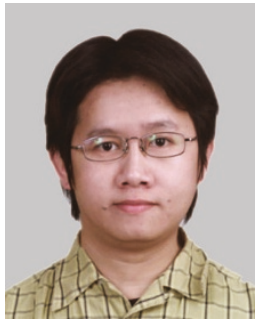

